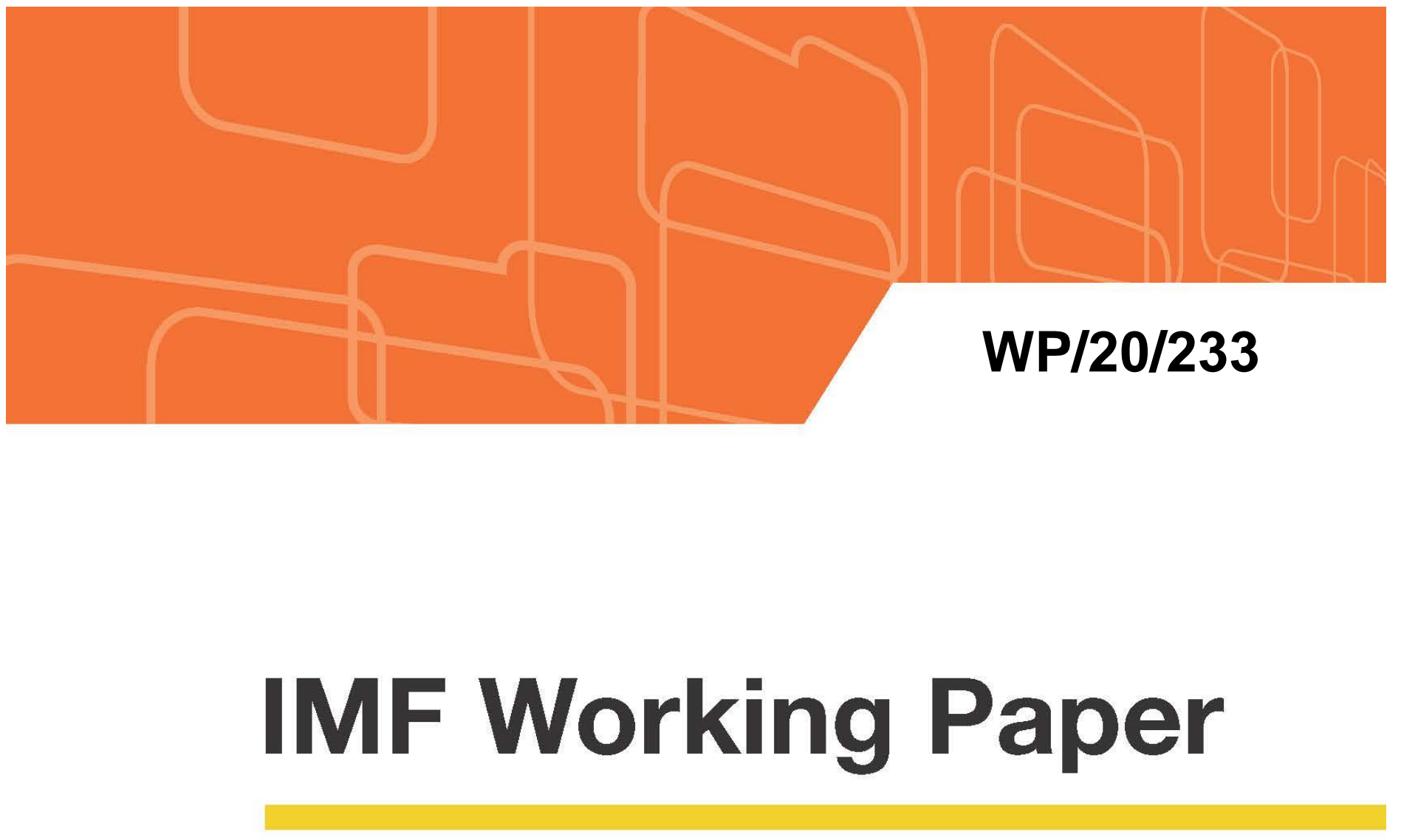

\title{
COVID-19 Impact and Mitigation Policies: A Didactic Epidemiological-Macroeconomic Model Approach
}

by John P. Ansah, Natan Epstein, and Valeriu Nalban

IMF Working Papers describe research in progress by the author(s) and are published to elicit comments and to encourage debate. The views expressed in IMF Working Papers are those of the author(s) and do not necessarily represent the views of the IMF, its Executive Board, or IMF management. 


\title{
IMF Working Paper
}

Institute for Capacity Development

\section{COVID-19 Impact and Mitigation Policies: A Didactic Epidemiological-Macroeconomic Model Approach}

\author{
Prepared by John P. Ansah, Natan Epstein, and Valeriu Nalban ${ }^{1}$ \\ Authorized for distribution by Stephan Danninger
}

November 2020

\begin{abstract}
IMF Working Papers describe research in progress by the author(s) and are published to elicit comments and to encourage debate. The views expressed in IMF Working Papers are those of the author(s) and do not necessarily represent the views of the IMF, its Executive Board, or IMF management.
\end{abstract}

\begin{abstract}
We develop an integrated epidemiological-macroeconomic model to analyze the interplay between the COVID-19 outbreak and economic activity, as a tool for capacity building purposes. We illustrate a workhorse framework that combines a rich epidemiological model with an economic block to shed light on the tradeoffs between saving lives and preserving economic outcomes under various mitigation policies and scenarios calibrated for emerging market and developing economies. In our benchmark setup, we link the effective contact frequency and labor supply decisions to the current state of the disease progression, allowing for relevant behavioral responses that introduce multiple feedback channels. We showcase the effects of various "smart" mitigation measures, e.g. improved quarantine capacity or targeted labor market restrictions, to alleviate the tradeoffs between health-related outcomes and economic activity, including in response to a second infection wave. The discovery of treatment or vaccine, and the possibility of temporary immunity for the recovered individuals are also considered. The model is further extended to a multisector framework to analyze the sectoral allocation effects of the COVID-19 shock.
\end{abstract}

JEL Classification Numbers: C63, E17, E61, H12, I18

Keywords: Pandemic; Epidemiological model; Macroeconomics; Emerging market and developing economies; Mitigation policy

Authors’E-Mail Addresses: john.ansah@duke-nus.edu.sg; nepstein@imf.org; vnalban@imf.org

\footnotetext{
1 The authors are grateful to Andrew Berg, Reda Cherif, Nada Choueiri, Gabriela Cugat, Stephan Danninger, Fuad Hasanov, Jochen Schmittmann and Nikola Spatafora for their thoughtful input and feedback, and to participants' comments on an earlier version presented at an IMF-ICD seminar.
} 


\section{Contents}

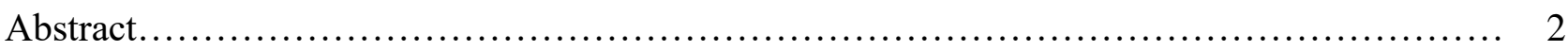

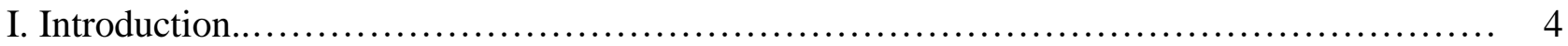

II. Integrated Epidemiological-Macroeconomic Model................................. 8

A. Epidemiological Block $\ldots \ldots \ldots \ldots \ldots \ldots \ldots \ldots \ldots \ldots \ldots \ldots \ldots \ldots \ldots \ldots \ldots \ldots \ldots \ldots \ldots \ldots, \quad 8$

B. Macroeconomic Block......................................................... 11

C. Model Calibration.............................................................. 12

D. Matching Epidemiological Data for Malaysia: An Illustration...................... 14

III. Mitigation Policies....................................................... 15

A. Lockdown Policy........................................................ 16

B. Enhanced Social Distancing .............................................. 18

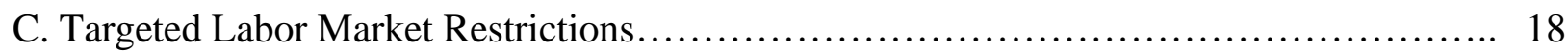

D. Improved Quarantine and Isolation........................................ 19

E. Increasing Telework Capacity ............................................. 19

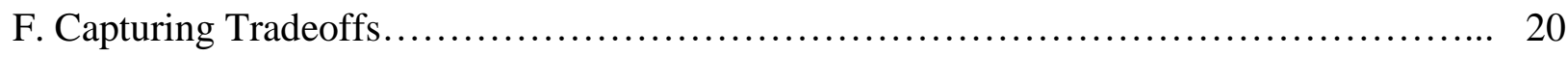

IV. Second Infection Wave, Medical Solutions and Temporary Immunity.................. 21

A. Second Infection Wave and Mitigation Policies ................................ 21

B. Medical Solutions ......................................................... 22

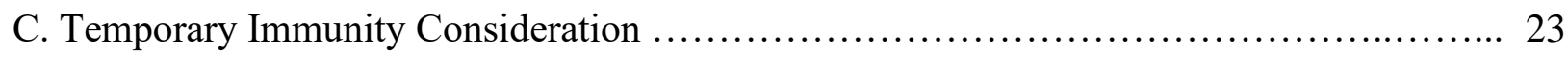

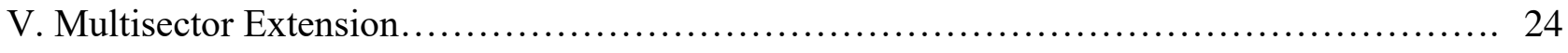

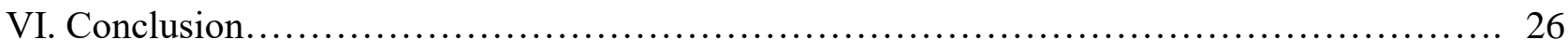

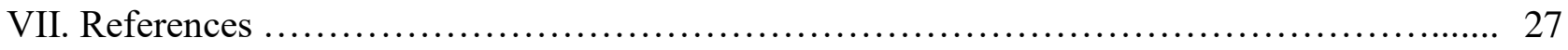




\section{INTRODUCTION}

The COVID-19 pandemic has affected - directly or indirectly - all countries and economic sectors. In order to contain the spread of the virus, countries adopted a wide range of non-pharmaceutical interventions (NPIs), like business stoppages, household lockdowns and travel restrictions. These containment measures were accompanied by large monetary and fiscal stimulus aimed at reducing the recessionary impact of the shock. Changes in social norms and consumption behavior are likely to persist even as lockdown measures wax and wane, and will likely last until proven vaccines and treatment become widely available.

These evolutions resulted in intricate feedback loops between the disease developments and economic activity, sparking a new literature stream attempting to integrate epidemiological and economic models. This new suite of models are used to analyze the complex interaction between the virus outbreak and economic activity, including to better understand the tradeoffs, explore the role of heterogeneities across various dimensions, simulate mitigation policies, and forecast the evolution of infection or macroeconomic variables.

The COVID-19 shock has generated multiple effects, with implications for different social and economic aspects. They include health-sector emergencies, supply-side disruptions, negative demand effects, financial turmoil, elevated uncertainty, etc. - making the analysis of the shock unique and unparalleled. In the emerging literature, empirical estimations use cross-country as well as regional-level survey-based data and highfrequency indicators to uncover the relation between business activity, labor markets, lockdown measures, population mobility, and the severity of the infection; see Chen et al. (2020), Cox et al. (2020), Deb et al. (2020), Goldberg and Reed (2020), Maloney and Taskin (2020) for preliminary results. Conclusions drawn based on previous pandemics, like the 1918 flu in Correia et al. (2020), while of limited comparability, are also used to understand the medium-to-long-run spillovers among NPIs, labor markets and economic activity.

The main objective of this paper is to shed light on the tradeoffs between saving lives and preserving economic outcomes under various mitigation policies and scenarios calibrated for emerging market and developing economies (EMDEs). The model is designed to capture relevant economic conditions and disease patterns typical in less developed countries, which have limited healthcare and financial resources, reduced telework capacity, insufficient precautionary savings and high levels of informality. We demonstrate that the framework can be easily calibrated to match specific country data to analyze a wide variety of scenarios and policies (e.g. a second infection wave, discovery of medical solutions, temporary immunity) in order to provide practical information for policymakers. The model also lends itself for training and capacity building purposes.

The main contributions and comparative advantages of our paper are: tractability, straightforward adaptation to particular country-cases, implementation of relevant policies and scenarios (including endogenous lockdown stringency, second infection wave and temporary immunity considerations), tradeoff assessments, and potential for model extensions (e.g. related to sectoral heterogeneity). These defining features of our framework make the model also suitable for capacity development - including at the level of authorities and government officials - as the model structure can be easily adapted to a specific country. In selecting the key features and assumptions of our model, we were guided by the emerging stylized facts and analyses in the literature, enumerated as follows:

The economic effects of the pandemic emerge quickly in EMDEs and their impact is substantial. Early indicators of the magnitude of the economic setback in EMDEs point to large output contractions. In addition, the plunge 
in commodity prices - particularly oil prices - is negatively affecting commodity-dependent countries. The durability of the economic downturns across countries remains difficult to assess but is clearly linked to disease developments. Even more so, compared to advanced economies (AEs), the EMDEs have underdeveloped healthcare systems, limited teleworking possibilities, and constrained ability to mobilize additional monetary or fiscal resources and efficiently deploy them. Generally warmer climate and younger population are possible epidemiological advantages EMDEs can enjoy. Goldberg and Reed (2020) show that deaths-per-capita depend positively on GDP-per-capita and negatively on the share of young population. ${ }^{2}$ On the other hand, Brussevich et al. (2020) estimate that workers in emerging economies have a lower index of feasibility to work from home and are at a higher risk of layoff.

Another relevant aspect is the voluntary behavioral response and the endogenous efficiency of lockdown measures in flattening the infection curve through curbing human interaction. The estimated results are obscured by the endogenous changes in behavior (e.g. wearing face masks, minimizing time spent outside, working from home), that would have surfaced even in the absence of government-mandated restrictions; both Chen et al. (2020) and Gupta et al. (2020) show that in many jurisdictions mobility decreased before the enactment of official lockdowns. Notwithstanding identification and endogeneity issues, estimations reveal that voluntary social distancing (proxied by the number of cases as an indicator of risk awareness, or using mobility data) appears to be more important than de jure measures, across both AEs and EMDEs: Chen et al. (2020) show that economic activity is driven by observed mobility, not by the imposition of de jure NPIs; Maloney and Taskin (2020) reveal that local and nationwide number of cases explain mobility trends better than NPIs; Goldberg and Reed (2020) obtain an insignificant correlation between the timing of lockdown measures and death rates; Alon et al. (2020) show that lockdowns are less effective in EMDEs, saving fewer lives per unit of lost GDP. These results also imply that the economic recovery is likely to be slow - once economies are officially open (i.e. de jure measures lifted), it will take time for the public to return to pre-COVID-19 habits.

A practical and feasible model also needs to account for the critical role of economic policies in managing the economic fallout. Economic stimulus deployed by the central banks and fiscal authorities have, at least partially, calmed financial markets, avoided credit market disruptions, limited firm exit and provided a safety net for affected individuals; their effectiveness in ensuring a sustained recovery and minimizing scarring effects remains to be seen. Unlike in AEs, fiscal space limits put a drag on government resource mobilization capacity in EMDEs. Higher prevalence of informality makes it harder to identify and provide support to individuals at risk; in addition, in such an environment the efficiency and compliance with de jure NPIs is lower, as empirically shown by Maloney and Taskin (2020). See also IMF (2020) and Cakmakli et al. (2020) for a discussion on policy responses in EMDEs, Loayza (2020) for tradeoffs faced by developing countries, KalemliOzkan (2020) for measures to support small business, and Brussevich et al. (2020) for demographic-related aspects.

Long-term implications in EMDEs are still too difficult to gauge. Most models have so far abstracted from making predictions regarding the long-run effects of the COVID-19 crisis, given the extremely uncertain evolution of the disease and of policy effects. Barrero et al. (2020) explore the emerging sectoral economic

\footnotetext{
${ }^{2}$ However, these outcomes are likely to be driven also by the insufficient testing and tracing in developing countries.
} 
restructuring and redistribution in terms of reallocation of jobs (e.g. out of high-contact industries, tourism, etc.), sales (increase in online commerce), working environment and business practices (toward teleworking), consumer spending behavior (out of high-proximity experiences like food away from home). While these shifts are mitigating infection risk - at least until a vaccine and an efficient treatment are available - the reallocation is not necessarily efficient from an economic perspective. Goldberg and Reed (2020) discuss the possible negative long-lasting economic and social effects: shifting toward online commerce can lead to increased monopoly power and lower competition; discontinuities in education processes are likely to affect human capital development, innovation activities and potential growth; attempts to re-nationalize supply chains can result in lower international trade, reversing the secular globalization trends. On the other hand, digitalization and higher work flexibility emerge as favorable outcomes of the pandemic. Overall, if these transformations materialize, the net negative repercussions are likely to be larger in EMDEs. ${ }^{3}$

Based on these considerations, our analytical framework builds on the standard SIR (susceptible, infected, recovered) epidemiological model developed by Kermack and McKendrick (1927). We extend it across several dimensions, including a two-way feedback loop between epidemiologic and macroeconomic blocks, allowing us to analyze a range of relevant mitigation policies. The epidemiological block matches various aspects of the disease and features asymptomatic infections and quarantine/isolation capacity. Baqaee et al. (2020) employ a similar structure, referring to it as a SEIRQD (susceptible, exposed, infected, recovered, quarantined, dead) framework. The economic block is represented by a log-linear production function in labor and total factor productivity (TFP), with the latter used to simulate investment in digitalization and teleworking equipment. The effect from the epidemiological block to economic activity is represented by the number of workers supplying labor services - taking into account potential lockdown restrictions imposed by the government. ${ }^{4}$ Similar to Eichenbaum et al. (2020), economic activity is impacting the pandemic progression through infections originating through consumption and at workplaces; we allow for the latter channel to be relatively more prevalent, matching the widespread informality in EMDEs, lack of precautionary savings for a large proportion of the population, and limited teleworking facilities. The parameterization follows the rich strand of emerging related literature, recognizing also the uncertainties regarding the biology of the virus and the natural history of the disease. Finally, the model does not provide economic predictions for the long-term impact of the COVID-19 shock.

In order to match the recent empirical evidence that the disease progression - as measured by new cases - is driving voluntary social distancing behavior and, therefore, economic outcomes, in our model the effective transmission rate is endogenous and determined by the current infection rate; equivalently, agents are assumed to experience disutility from infection risks. This relationship is also embedded in other theoretical frameworks: agents' health declines with the contact frequency in Gupta et al. (2020); the share of voluntary isolated agents depends on the probability of contracting the virus in Chudik et al. (2020); contact frequency is adjusted by the

\footnotetext{
${ }^{3}$ Even if an effective medical solution to COVID-19 is found, the mentioned effects can still persist as a precautionary response to possible future epidemics.

${ }^{4}$ We do not explore the optimal lockdown policies, as done in Acemoglu et al. (2020) or Alvarez et al. (2020). This allows us to preserve tractability of the analytical tool and avoid the use of controversial assumptions, like the pecuniary "value of a statistical life", necessary to perform such theoretical exercises.
} 
current proportion of infected ("short-term awareness") or cumulative proportion of affected individuals ("longterm awareness") in purely-epidemiological simulations of Eksin et al. (2019); and the magnitude of demandside shocks is determined by the infection rate in Cakmakli et al. (2020). We also simulate alternative endogenous behavioral responses through linking the efficiency of the lockdown measures to the current economic developments, such that as the recession becomes more severe, the compliance with the restrictions declines; Maloney and Taskin (2020) find that, in low income countries, mobility declines are not significantly affected by NPIs, citing low enforcement of and high resistance to the de jure restrictions.

We simulate a range of mitigation policy scenarios: increased social distancing that reduces effective contact frequency (e.g. wearing masks, keeping physical distance, thorough personal hygiene); targeted labor market restrictions (widespread testing and better contact tracing allows to identify a larger share of infectious, including asymptomatic, restricting their access to workplaces); increased identification and quarantine capacity that reduces the average delay between contracting the virus and identification; and investment in teleworking capacity. The effectiveness of these measures is also studied in a second infection wave scenario, similar to Baqaee et al. (2020).

We find that all these measures manage to flatten both infection and recessionary curves; however relative improvements of health versus economic outcomes vary across policies. Following Acemoglu et al. (2020), we represent the mitigation of this tradeoff between lives (death rate) and livelihoods (output drop) using the pandemic possibility frontier (PPF). The discovery of effective vaccines or medical treatment, as well as, the yet-uncertain possibility of only temporary immunity for the recovered individuals is also discussed.

Finally, we explore the role of production sector heterogeneities within a multisector extension of the model, assuming there are affected/non-essential and unaffected/essential economic activities, in line with the early empirical results of Barrero et al. (2020) and following the theoretical framework of Krueger et al. (2020). We find that the aggregate impact - both in terms of health and macroeconomic outcomes - depends on the relative weight of and the degree of substitutability between sectoral goods. For example, easier substitution allows sectoral reallocation and cushions the epidemic shock in aggregate terms, but this buffer can be limited in case the affected sector is large. Other important heterogeneities - e.g. age-specific fatality rates and interaction patterns, gender-related differences, occupation-specific flexibility and propensity to telework - are left for future research; see related modelling frameworks in Acemoglu et al. (2020), Alon et al. (2020), Baqaee et al. (2020), Cakmakli et al. (2020), Krueger et al. (2020). ${ }^{5}$ In the present paper, our contribution refers to developing a tractable, yet realistic, framework to simulate the outcomes across stylized representations of the various mitigation measures implemented by policymakers. The current structure can be extended to overcome some of the listed challenges.

This paper is organized as follows. Section 2 provides a description of the model and its parameterization. Section 3 presents the main results across various mitigation policy scenarios. In Section 4 we present additional

\footnotetext{
${ }^{5}$ Among other relevant limitations of the present framework, especially with respect to the modelled economic structure are: abstraction from capital services as a production factor, no explicit household tradeoff between consumption and savings or between consumption and labor, no external sector (open economy) considerations, absence of detailed monetary and fiscal policy transmission.
} 
simulations for the case of the second infection wave, the impact of mitigation policies and the arrival of medical treatment or vaccine. Section 5 showcases the multisector extension, while Section 6 concludes.

\section{INTEGRATED EPIDEMIOLOGICAL-MACROECONOMIC MODEL}

Our model is an extension of the seminal SIR (susceptible, infected, recovered) framework of Kermack and McKendrick (1927). The baseline setting resembles the SEIRQD (susceptible, exposed, infected, recovered, quarantined, dead) structure of Baqaee et al. (2020). The time frequency of the model is daily. We differentiate between diagnosed and undiagnosed infected individuals. The former comprise reported (positive-tested and documented) cases, while the latter represent the unobserved share of the population who is infected but not identified; the true extent of the infection is thus likely to be severely underestimated in countries with insufficient testing capacity - particularly EMDEs - where the number of undiagnosed individuals is prone to be large. The rich epidemiological model allows us to simulate relevant and specific mitigation policies in order to uncover the range of health versus economic tradeoffs imposed by the COVID-19 crisis.

\section{A. Epidemiological Block}

The health states in the model are: susceptible $(S)$ - individuals at risk of becoming infected; undiagnosed infected latent $(U I L)$ - infected individuals that are not diagnosed yet and do not have enough viral load to infect other people (additionally, they do not present symptoms, since the scientific evidence points to the onset of symptoms arriving after the onset of infectiousness; see He et al. (2020)); undiagnosed infected infectious (UII), who are not identified but have developed enough viral load to infect other persons (while some of these present symptoms, others are still asymptomatic, matching the evidence that a significant proportion of transmissions is asymptomatic); undiagnosed infected non-infectious (UINI) - individuals at the latter stage of the infection where they cannot infect others because of insufficient viral load; diagnosed infected quarantined/isolated $(Q)$, consisting of infected individuals (latent, infectious, or non-infectious) who are diagnosed via testing and isolated from society; recovered $(R)$; dead $(D)$. Thus, the total population $(T)$ is:

$$
T(t)=S(t)+U I L(t)+U I I(t)+U I N I(t)+Q(t)+R(t)+D(t)
$$

The epidemic dynamics is depicted in Figure 1. Through exposure of susceptible individuals $(S)$ to an infected infectious individual (UII) - via work, consumption and/or random meetings, following Eichenbaum et al. (2020) - some of them contract the virus and become infected. Infected individuals go through three infection stages: latent (and asymptomatic), infectious (a mix of asymptomatic and symptomatic individuals) and noninfectious. At any of these, if tested positive and diagnosed, the individual is placed into quarantine/isolation $(Q)$. Undiagnosed latent $(U I L)$ is the infection stage during which the person cannot infect others yet; its length is determined by a specific latency period (or onset of infectiousness). All newly infected individuals will transition over time from the undiagnosed latent health state to either undiagnosed infectious (UII), when they can spread the infection further; or be diagnosed via testing and isolated from society into quarantine $(Q)$. Over time, undiagnosed infectious individuals will either die $(D)$ from the disease, or be diagnosed via testing and put into quarantine $(Q)$, or will pass through the undiagnosed non-infectious state (UINI) before surviving the virus and fully recover $(R)$. Once diagnosed and quarantined/isolated $(Q)$, one part of individuals will recover 
$(R)$ from the disease, while the rest is expected to die $(D)$ due to infection complications and comorbidities. In our model we assume that only undiagnosed infected infectious (UII) transmit the virus; note that given the latency period is lower than the incubation period, some of these individuals are asymptomatic. Due to proper isolation and compliance to strict rules, diagnosed infectious individuals placed into quarantine do not create new infections. The equations that describe the evolution of the population in each health state over time are described below.

Figure 1: Epidemiological block

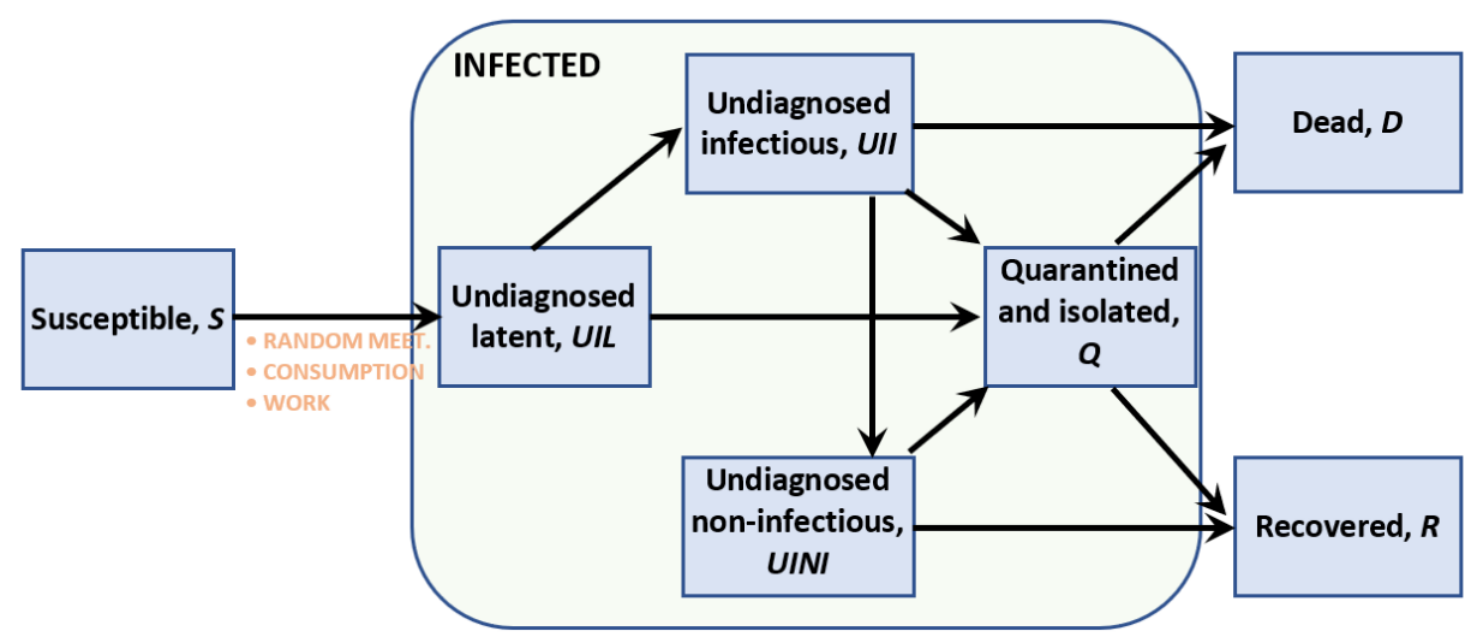

There are three possible infection sources: labor $(L)$, consumption $(C)$, and random meetings $(R M)$. Let $j$ denote these, i.e. $j \in\{L, C, R M\}$. Then, the flow of new infections determines the change in the susceptible pool:

$$
\Delta S(t)=-\beta(t) \gamma(t) \sum_{j} \omega_{j} \alpha_{j}(t) \cdot[1-\kappa \cdot \tau(t)]
$$

$\beta$ is the probability of infection given a contact between susceptible and infected individuals, while $\gamma$ is the contact frequency; both are allowed to vary over time, reflecting the imposition of lockdown measures and/or changes in individual social distancing behavior. $\omega_{j}$ are used to calibrate the relative shares of infections' originations. $\alpha_{j}$ reflects the modality of the contact between infected and susceptible individuals, disaggregated across activities: for workplaces $(j=L)$ it is determined by the labor fraction of susceptible and undiagnosed infectious workers who are allowed to access the labor market, $L_{S} \cdot \frac{L_{U I I}}{L_{T}}$; for consumption $(j=C)$ it is proportional to the share of total output $(Y)$ consumed by undiagnosed infectious population, $\frac{U I I}{T} \cdot Y$; for random meetings $(j=R M)$, similarly to the benchmark SIR model, it is the product of the susceptible pool and the population share of undiagnosed infectious individuals, $S \cdot \frac{U I I}{T}$. Note that we assumed herein that diagnosed infected individuals $(Q)$ are fully compliant with strict isolation rules and do not create infections.

Unlike standard SIR models, in our baseline specification the number of new infections is also determined by the behavioral response of the population internalizing the infection risk and the "disutility" it induces. The 
term $[1-\kappa \cdot \tau(t)]$, where $\tau(t)$ is the current fraction of infected population, $\tau(t)=\frac{U I L(t)+U I I(t)+U I N I(t)+Q(t)}{T(t)}$, represents the reduction in virus transmission in case of additional voluntary social distancing - possibly in the absence of any de jure measures - as determined by the sensitivity parameter $\kappa$. This extension of the model is in line with the empirically documented relation between the decline in mobility and the number of reported COVID-19 cases in Chen et al. (2020) and Maloney and Taskin (2020). Our approach is also isomorphic to other similar mechanisms considered in the models of Gupta et al. (2020), Chudik et al. (2020), Eksin et al. (2019) or Cakmakli et al. (2020).

The evolution of undiagnosed infected latent (UIL) is determined by new infections, less the individuals who have developed enough viral load to spread the virus further and became infectious, and those who have been tested positive and put in isolation:

$$
\Delta U I L(t)=\beta(t) \gamma(t) \sum_{j} \omega_{j} \alpha_{j}(t) \cdot[1-\kappa \cdot \tau(t)]-\frac{U I L(t)}{\chi}-\phi(t) \frac{U I L(t)}{\delta}
$$

In equation (3), $\chi$ denotes the latency period (number of days between contracting the virus and becoming infectious); $\delta$ represents the isolation delay, i.e. the average period to identify an infected person; $\phi(t)$ is the time varying quarantine fraction, evolving according to $\phi(t)=\phi_{0} \cdot\left[1-\phi_{1} \cdot \tau(t)\right]$. While $\chi$ is a parameter specific to the disease biology, $\delta, \phi_{0}$ and $\phi_{1}$ are policy parameters reflecting available testing volume, contact tracing performance and quarantine/isolation capacity, which are likely to be lower in EMDEs as compared to AEs.

The equation for undiagnosed infected infectious (UII) is:

$$
\Delta U I I(t)=\frac{U I L(t)}{\chi}+M(t)-\phi(t) \frac{U I I(t)}{\delta}-\frac{U I I(t)}{\sigma}-\rho U I I(t)
$$

The inflow (first two terms) reflects individuals transitioning out of latency and possible imported cases $M$, i.e. infected persons arriving from abroad. Outflows (last three terms) consist of individuals who are identified and quarantined, infectious becoming non-infectious, and deaths. $\sigma$ is a constant parameter reflecting the period of infectiousness after which the viral load is insufficient to transmit the virus to others, while $\rho$ is mortality rate.

Undiagnosed infected non-infectious (UINI) evolve according to:

$$
\Delta U I N I(t)=\frac{U I I(t)}{\sigma}-\phi(t) \frac{U I N I(t)}{\delta}-\psi U I N I(t)
$$

Equation (5) represents inflows based on infected individuals who are no longer infectious (first term), less the identified cases who are put into quarantine (second term) and those who recover (last term), given the recovery rate $\psi$.

The pool of diagnosed quarantined/isolated $(Q)$ reflects new arrivals from unidentified latent, infectious, and non-infectious states, less dead and recovered:

$$
\Delta Q(t)=\phi(t) \frac{U I L(t)+U I I(t)+U I N I(t)}{\delta}-\rho Q(t)-\psi Q(t)
$$

The numbers of recovered (R) and dead (D) individuals are determined as: 


$$
\begin{aligned}
& \Delta R(t)=\psi U I N I(t)+\psi Q(t) \\
& \Delta D(t)=\rho U I I(t)+\rho Q(t)
\end{aligned}
$$

\section{B. Macroeconomic Block}

The spillover from the pandemic to economic activity manifests itself in reductions of labor supply, which lead to proportionally lower output $(Y)$ under the assumed log-linear production function technology. Following the relevant literature, we abstract from other production factors, like capital services. With this simplification, we recognize the primacy of the labor input in determining output under social distancing measures, avoiding also modeling intertemporal decisions for the households, which would complicate numerical simulations. Total labor input $(L)$ in terms of number of workers (extensive margin) is the sum of labor supplied by each of the health groups, $i \in\{S, U I L, U I I, U I N I, Q, R, D\}$. The latter is computed as the total group population adjusted

by: working age population share, $\frac{W A P_{i}}{i}=$ washare $_{i}$; employment rate, $\frac{L_{i}}{W A P_{i}}=$ emplrate $_{i}$; share of persons in group $i$ supplying labor services, sharework $_{i}$; and the voluntary social distancing response, $[1-\kappa \cdot \tau(t)]$ :

$$
\begin{gathered}
Y(t)=Z(t) L(t) \\
=Z(t) \sum_{i} i(t) \cdot \frac{W A P_{i}(t)}{i(t)} \cdot \frac{L_{i}(t)}{W A P_{i}(t)} \cdot \operatorname{sharework}_{i}(t) \cdot[1-\kappa \cdot \tau(t)] \\
=Z(t) \sum_{i} i(t) \cdot \text { washare }_{i}(t) \cdot \text { emplrate }_{i}(t) \cdot \operatorname{sharework}_{i}(t) \cdot[1-\kappa \cdot \tau(t)]
\end{gathered}
$$

Production function (9) allows also for the presence of a total factor productivity term, $Z$, which will be used to simulate investment in digital equipment to facilitate teleworking. sharework $k_{i}$ is used to simulate targeted labor market restrictions ("smart containment"); e.g. under more efficient testing and tracing the authorities can identify the undiagnosed infectious individuals (UII) and forbid their access to the labor market, thus reducing the infection rate at workplaces.

Government-mandated lockdowns play a critical role in curbing the spread of infections during the epidemic. In an alternative formulation, we shut down the voluntary social distancing behavior $(\kappa=0)$ and append to the new infections term in (1)-(2) and the production function (9) a time-varying indicator approximating the impact of de jure restrictions on labor supply, Lockdown. Similar to Alvarez et al. (2020) and Acemoglu et al. (2020), the lockdown is defined as the share of the population that is subject to stay-at-home policy, $P$, adjusted with an efficiency factor $\theta$ :

$$
\operatorname{Lockdown}(t)=[1-\theta(t) \cdot P(t)]
$$

A more extensive (higher $P$ ) and/or more stringent (higher $\theta$ ) lockdown reduces the spread of the virus but has negative impact on output, creating a tradeoff between saving lives and preserving economic outcomes. Time variation in the efficiency parameter $\theta$ is used to simulate reduced compliance with the de jure lockdown measures as the economic situation worsens, which is especially relevant for EMDEs. 


\section{Model Calibration}

The scientific community is still learning about the biology of SARS-CoV-2, implying large uncertainties around model parameters. We follow the literature and consider generic values for most of the parameters. In the case of several specific parameters, we use relevant economic and epidemiological data from Malaysia. The choice of Malaysia as a representative EMDE is motivated by several relevant structural economic and social indicators that are numerically close to the group's average. Malaysia is a medium-size country in terms of population (32.4 million in 2018) and economy (nominal GDP of US\$364 billion in 2019), with a development level close to the global average (GDP per capita of around US\$11 thousand in 2019). It is a very open economy (sum of imports and exports exceeds GDP), with the main exporting categories including both electrics and electronics (38 percent of total exports in 2018) - which are representative for (mostly Asian) emerging economies - and commodities, including petroleum (23 percent) - which are particularly representative for developing economies. ${ }^{6}$ See also the next subsection where we showcase how the model can be used in conjunction with the reported COVID-19 cases to estimate some of the parameters.

Following the structure of the integrated model, parameters can be divided into two groups: those specific to the epidemiological block and the economic parameters, respectively. Table 1 displays the calibration of the epidemiological block. Most of the parameters are within the range of values used in the relevant literature. Based on evidence from He et al. (2020) and NCID (2020), latency period $(\chi)$ is set to 4 days and duration of infectiousness $(\sigma)$ is fixed at 10 days. The transition period from non-infectiousness state to recovery (given by the inverse of $\psi$ ) is calibrated at 21 days. ${ }^{7}$ Mortality rate $(\rho)$ is set at a lower range of the values considered in the literature, given our mapping to EMDEs; this is in line with the cross-country empirical evidence of positive correlation between deaths and income found in Goldberg and Reed (2020). Probability of infection given $\operatorname{contact}(\beta)$ is set to 15 percent, while contact frequency $(\gamma)$ is assumed to drop from the average 5 persons per day to 2 persons per day once the government declares emergency state (at day 58 in Malaysia), thus reflecting the changes in social behavior observed across populations. These values are in line with the contact matrices illustrated in Baqaee et al. (2020) before and after the COVID-19 outbreak.

Isolation/quarantine fraction is assumed to be determined by the initial capacity to quarantine 5 percent of the previously undiagnosed infected, taking an average period of 5 days to identify them. Eichenbaum et al. (2020) calibrate the shares of infection origins (implied by $\omega_{i}$ ) for the USA to $1 / 6$ from consumption, 1/6 from work, and 4/6 from random interactions. Given our focus on EMDEs - with pervasive labor informality, minimal teleworking, and limited precautionary savings - we double the share of the workplace-related infections at the expense of the random meetings' share.

\footnotetext{
${ }^{6}$ The availability and reliability of the epidemiological data required for the data matching exercise were also important considerations for choosing Malaysia as a representative country.

${ }^{7}$ This implies that it takes on average 35 days (4 days of latency plus 10 days of infectiousness plus 21 days until full recovery) between contracting the virus and returning to the labor market. As mentioned in Baqaee et al. (2020), the range of values for this parameter is very wide, including because of heterogenous hospital policies across jurisdictions regarding the discharge of recovered COVID-19 patients.
} 
Table 1: Model calibration: Epidemiological block

\begin{tabular}{lll}
\hline Notation & Value & Description \\
\hline$\chi$ & 4 & Latency period \\
$\sigma$ & 10 & Duration of infectiousness \\
$\psi$ & $1 / 21$ & Recovery rate \\
$\rho$ & 0.0025 & Mortality rate \\
$\beta$ & 0.15 & Probability of infection given contact \\
$\gamma(t \mid t<58)$ & 5 & Contact frequency before emergency state declared \\
$\gamma(t \mid t \geq 58)$ & 2 & Contact frequency after emergency state declared \\
$\delta$ & 5 & Isolation delay \\
$\phi_{0}$ & 0.05 & Quarantine fraction under no infection \\
$\phi_{1}$ & 10 & Quarantine fraction sensitivity to infection rate \\
$\omega_{C}$ & 0.17 & New infections share from consumption activities \\
$\omega_{L}$ & 0.33 & New infections share from labor activities \\
$\omega_{R M}$ & 0.5 & New infections share from random meetings \\
\hline
\end{tabular}

A second group of parameters is related to the macroeconomic block; see Table 2. For simplicity, we assume that working age share and employment rate are constant across the health groups and across time. For Malaysia these represent 69.6 percent and 66.8 percent in 2018, respectively (based on Department of Statistics Malaysia data). ${ }^{8}$ The proportion of persons across health groups who are assumed to work, sharework $k_{i}$, can be calibrated by approximating sector-specific NPIs, or the social behavior of workers who may decide to isolate (voluntary lockdown) and/or work from home. In the baseline setting we assume that (i) all susceptible, unidentified latent, and recovered work (because, respectively, they are not sick, they are asymptomatic and not infectious yet, and they had already recovered); (ii) only 50 percent of undiagnosed infectious and non-infectious work (i.e., even if sick, 50 percent are not identified - including because some of them are asymptomatic - and continue supplying labor services); (iii) quarantined are restricted from the labor market.

Table 2: Model calibration: Macroeconomic block

\begin{tabular}{lll}
\hline Notation & Value & Description \\
\hline washare $_{i}$ & 0.696 & Share of working age population \\
emplrate $_{i}$ & 0.668 & Employment rate \\
sharework $_{i}$ & 1 & Share of workers, $i \in\{S, U I L, R\}$ \\
sharework $_{i}$ & 0.5 & Share of workers, $i \in\{U I I, U I N I\}$ \\
sharework $_{i}$ & 0 & Share of workers, $i \in\{Q, D\}$ \\
$\kappa$ & $1 / 0$ & Behavioral response (baseline / if $\theta=0.75)$ \\
$\theta$ & $0 / 0.75$ & Lockdown efficiency (baseline / if $\kappa=0)$ \\
\hline
\end{tabular}

\footnotetext{
${ }^{8}$ Given the generally younger population of EMDEs and the evidence that COVID-19 has different effects across age groups, keeping the working age population share constant in the model is likely to overestimate the prevalence of the infections and deaths.
} 
The sensitivity of contact frequency to infection risk, $\kappa$, is set to 1 ; given it is a non-standard parameter, we perform a sensitivity analysis in the next section. In our baseline calibration, when $\kappa=1$, we assume no active lockdown policy, i.e. $\theta=0$, under the assumption that the impact of the government-mandated restrictions is already captured by the behavioral changes under $\kappa=1$. In the alternative calibration, when no such changes occur $(\kappa=0)$ and the lockdown measures are in place, we assume an efficiency parameter $\theta=0.75$, as in Acemoglu et al. (2020). This lockdown efficiency is within the range defined by the value of 0.5 used in Alvarez et al. (2020) and the "community understanding" parameter of 0.9 computed in the Oxford COVID-19 Government Response Tracker for the Malaysia Stringency Index. We provide a sensitivity analysis for this parameter, assessing the corresponding tradeoff it implies. ${ }^{9}$

\section{Matching Epidemiological Data for Malaysia: An Illustration}

While we use a generic calibration to showcase the main simulation results, the modelling framework can also be flexibly used to match specific country data and provide more practical information for policymakers. As an application, we use reported epidemiological data for Malaysia between late-January to early-July 2020 (source: Malaysia Ministry of Health) - new confirmed and imported cases, recovered individuals and deaths - to employ a matching procedure by numerically iterating over the values of several parameters. ${ }^{10}$ The results are presented in Figure 2.

Figure 2: Matching Malaysia data
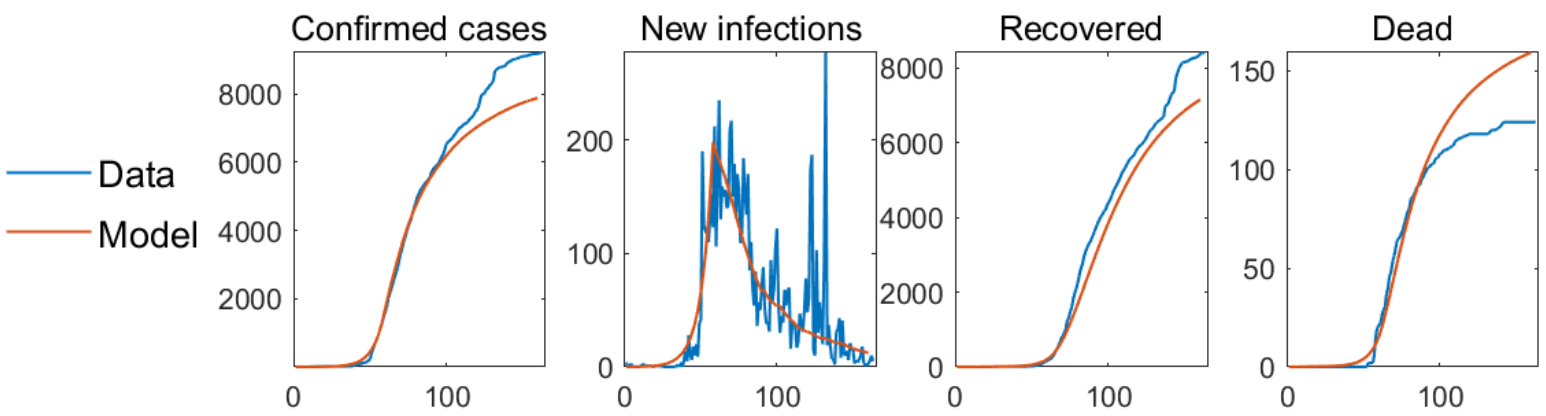

\footnotetext{
${ }^{9}$ Both the sensitivity parameter $\kappa$ and the lockdown efficiency $\theta$ can be anchored to (i) the Oxford COVID-19 Government Response Tracker (OxCGRT) "stringency index", which records the strictness of lockdown policies implemented by the governments to contain people's behavior (e.g. school closures, workplace closures, travel bans); and (ii) population mobility data provided by multinational technology companies.

${ }^{10}$ Parameter changes with respect to the baseline calibration in Table 1 are: recovery duration of 18 days, isolation delay of 10 days, constant fraction quarantined of 60 percent, (estimated) contact frequency of 4.5069 persons, (estimated) probability of infection given contact of 0.1678 .
} 
The model matches COVID-19 cases remarkably well, especially during the initial period (first three months). Given the (seemingly random) one-day spikes in new infections around days 120-130 (late-May to early-June), the matching of cumulative measures (cases, recoveries, deaths) worsens around that moment. In order to approximate the new slopes better, the matching procedure can be extended to allow for parameter break points (more generally, time variation in parameter values), a topic left for future research.

\section{Mitigation Policies}

We present our simulation results in terms of the time paths of the infection rate (population share of infected), total (cumulative) infection rate, total (cumulative) share of deaths, and output deviation from the pre-pandemic value. Our flexible analytical framework encompasses the standard (extended) SIR model, obtained under no endogenous behavioral responses and no lockdown policy effects - i.e. by calibrating $\kappa=\theta=0$. We refer to this simulation as "reference epidemiological model"; see Figure 3. By the end of one year, about 1/3 of the population is infected, with deaths representing about 0.8 percent of the population. ${ }^{11}$ The economic costs represent an average annual GDP loss of about 2.2 percent.

Figure 3: Reference model (no behavioral response, no lockdown)

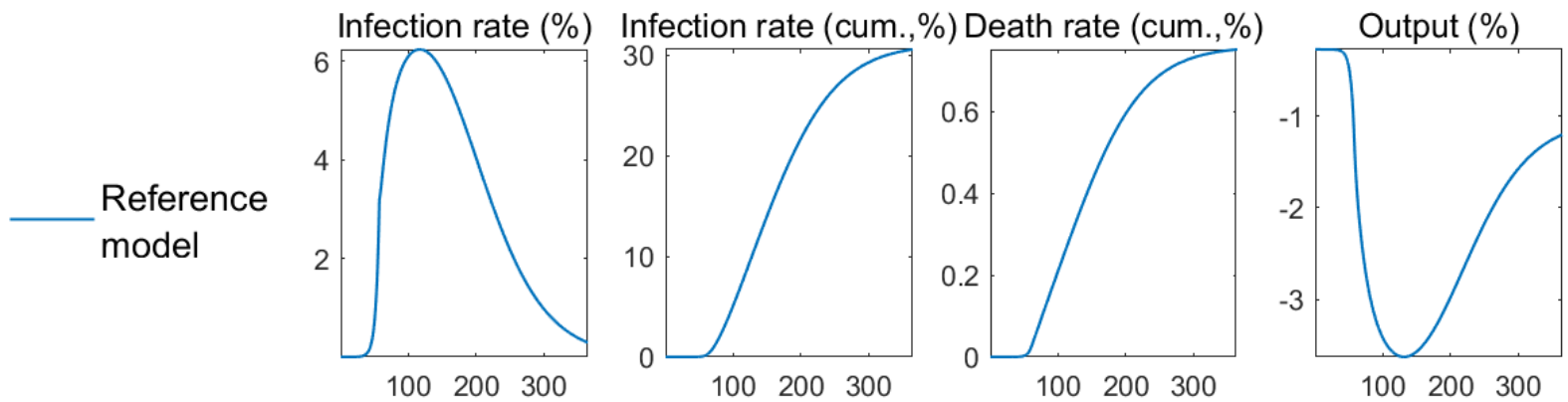

The results for our baseline model, with behavioral response $(\kappa=1)$ but no lockdown policy $(\theta=0)$, are presented in Figure 4. We also show the alternative simulations with higher and lower sensitivity of the effective contact frequency to the infection risk. Voluntary social distancing reduces the spread of the virus, with less infections and deaths as compared to the reference model, but at the cost of steeper output contraction. In particular, for the benchmark calibration $(\kappa=1)$ the annual average GDP loss amounts to 4.8 percent, with the sharpest drop in the third and fourth months after the first COVID-19 case. Note that our calibration implies an exponential increase of infections and an abrupt output decrease once the spread of the virus accelerates; then,

\footnotetext{
${ }^{11}$ In line with most of the literature on integrated epidemiological-macroeconomic models, we interpret the health-related outcomes in terms of "true" infection prevalence; see Acemoglu et al. (2020), Alvarez et al. (2020), Eichenbaum et al. (2020), Krueger et al. (2020). In practice, officially reported data for any country - which is significantly lower than typical model-based results - is likely to underestimate the true extent of the pandemic because of insufficient testing. This issue is particularly relevant for EMDEs.
} 
after peaking at around 15 weeks, both new infections and output slowly return to equilibrium. This outcome is in line with the available high frequency statistical data, as documented in the emerging empirical literature; see Chen et al. (2020), Cox et al. (2020), Deb et al. (2020), Goldberg and Reed (2020), Maloney and Taskin (2020).

Figure 4: Baseline model (behavioral response)

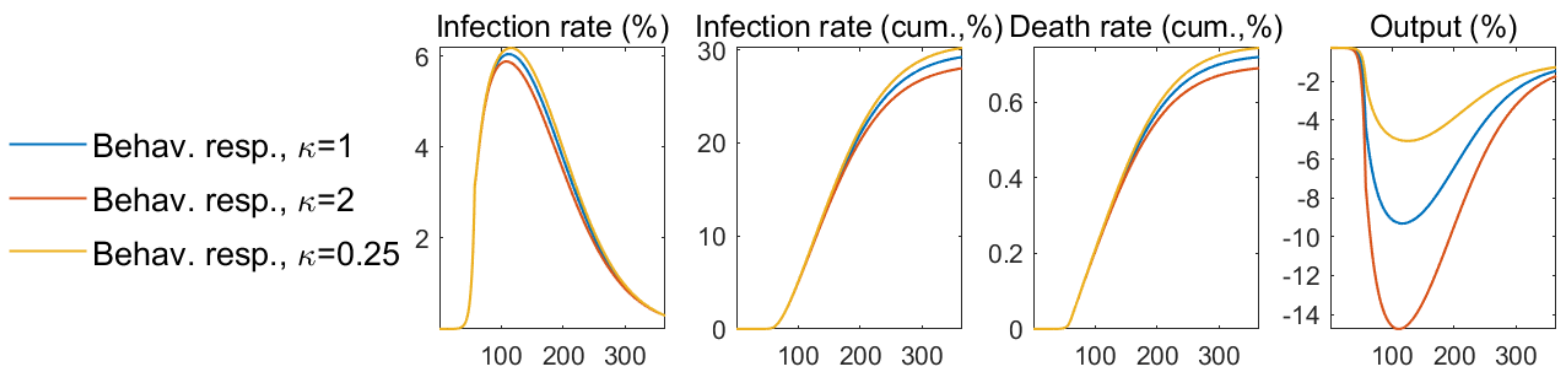

\section{A. Lockdown Policy}

The implementation of the lockdown policy simulations in our framework is based on the exogenous path of the share of restricted interactions, as specified in equation (10). Following the optimal policy results in Acemoglu et al. (2020) and Alvarez et al. (2020), we specify a trajectory for the policy variable $P(t)$ featuring an abrupt increase to about 20 percent of the population, followed by a slow return by the end of the simulation interval; see Figure 5. This gradual dynamic is intended to approximate the observed slow recovery in population mobility even after some of the de jure restrictions were lifted.

Figure 5: Time path of lockdown policy

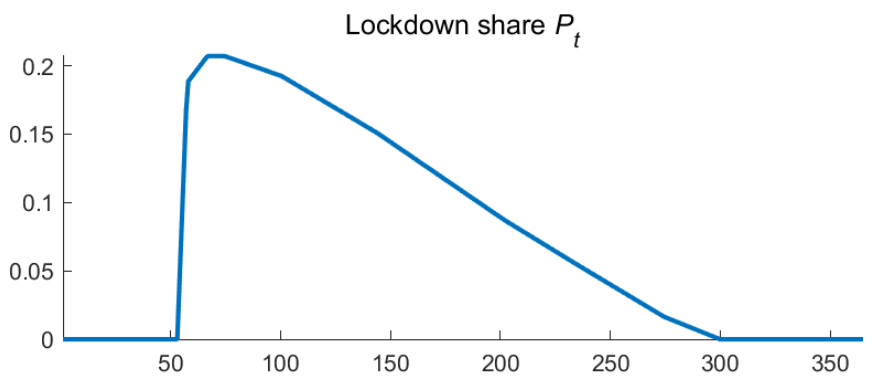

Simulation results for the alternative model with lockdown policy are presented in Figure 6. In the simulation with the efficiency parameter $\theta=0.75$, the infection prevalence and death rate are reduced to 23 percent and 0.6 percent, respectively. At the same time, output declines by 9 percent during the year. Sensitivity analysis with respect to the lockdown efficiency reflects the tradeoff between health outcomes and economic activity: 
in case of lower compliance with the lockdown measures (low $\theta$ ), the recession curve is flatter by about 5 percentage points at the through, but at the cost of higher prevalence of the disease and morality rate.

Figure 6: Alternative model: lockdown policy

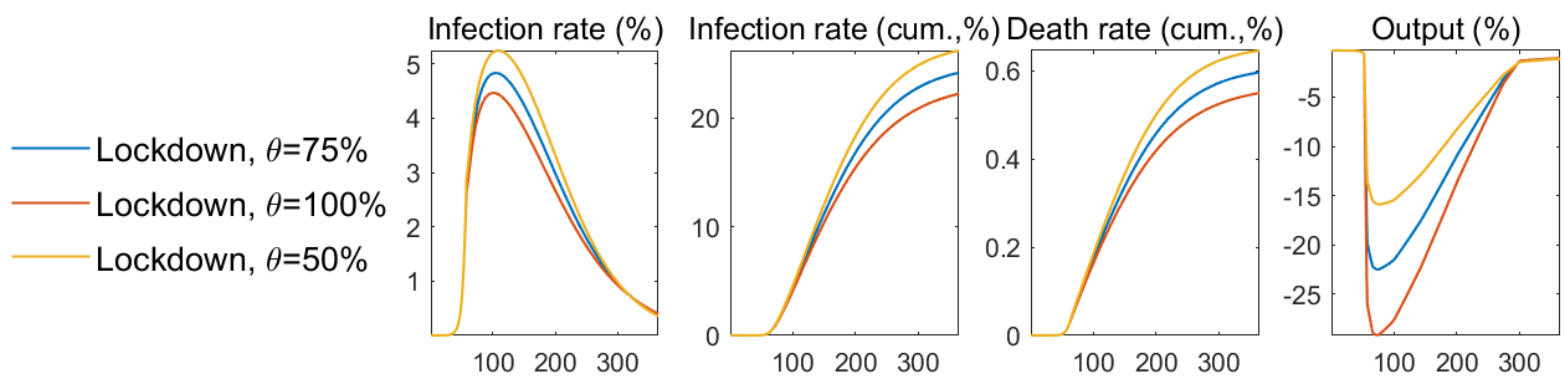

A relevant extension of the lockdown model - especially for EMDEs - is related to endogenizing the efficiency parameter $\theta$ by linking it to the current economic developments (percent GDP growth relative to pre-pandemic value, $\Delta \% Y(t)=Y(t) / Y(0) \cdot 100-100)$ :

$$
\theta(t)=\theta \cdot[1+\mu \cdot \Delta \% Y(t)]
$$

This relation reflects how compliance with the lockdown orders decreases proportionally with the severity of economic contraction. In EMDEs - with higher labor informality, liquidity-constrained population, limited teleworking and insufficient precautionary savings - the sensitivity of lockdown efficiency to output decline is likely to be significant ( $\mu$ high); Alon et al. (2020) consider these particularities in their incomplete-markets macroeconomic model with epidemiological dynamics. Figure 7 shows the simulations across different values of $\mu$. The relative improvement in economic gains as the sensitivity parameter $\mu$ increases is matched by a cost in terms of health outcomes. For example, in the simulation with $\mu=3$, which represents an economy with low compliance with the de jure NPIs once the economic crisis arrives, average output loss during the first year is about 1 percentage point lower as compared to the simulation with $\mu=1$, while the population share of deaths is 0.03 percentage points higher. Overall, output appears to be relatively more responsive to the lockdown sensitivity to infection rate as compared to health sector indicators. Given the simplicity of the macroeconomic block, the lockdown policy has a direct impact on production; at the same time, the richer interactions among the various health groups within the epidemiological block makes the lockdown impact weaker (e.g. under lockdown the pool of susceptible is larger, allowing, ceteris paribus, for a higher probability of infection given the specific value of the partial lockdown sensitivity to current infection rate). Additionally, in the simulations with endogenous lockdown efficiency we turn off the voluntary social distancing channel $(\kappa=0)$, which minimizes the incidence of the disease. 
Figure 7: Alternative behavior: endogenous lockdown efficiency
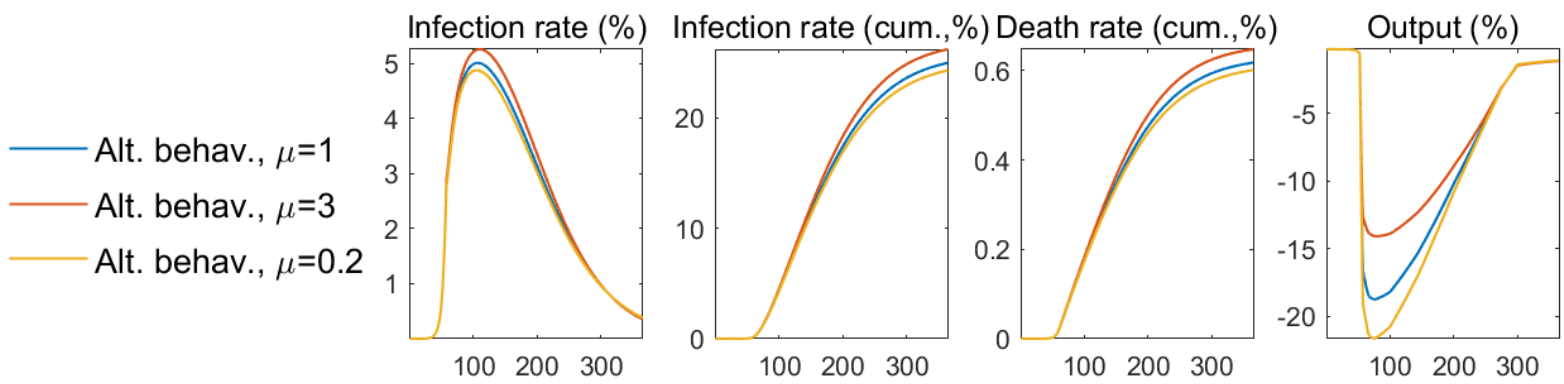

\section{B. Enhanced Social Distancing}

The first mitigation policy we analyze relates to better social distancing, achieved through specific recommendations and measures regarding wearing facemasks, physical distancing, eye protection, restrictions on gatherings, identification of risk groups, public disclosure of infected cases, etc. Chu et al. (2020) provide a comprehensive meta-analysis on the marginal effects of individual protection measures in preventing personto-person transmission of SARS-Cov-2. Since in our model contact frequency and probability of infection given contact enter multiplicatively, we perform and interpret the corresponding simulation in terms of reducing direct virus transmission rate. Technically, we assume that the additional measures reduce effective contact frequency by 15 percent as compared to the baseline calibration (from 2 to 1.7 persons per day for $t \geq 58$ ). Simulation results in Figure 8 show the improvements achieved under this policy. The total infection rate reduces to only 10 percent of the population, death rate accounts for 0.25 percent of the population, while GDP declines by an annual average of less than 2 percent. Accordingly, reducing effective contact frequency proves to be an efficient policy in mitigating the tradeoffs between economic activity and health outcomes.

Figure 8: Mitigation policy: better social distancing

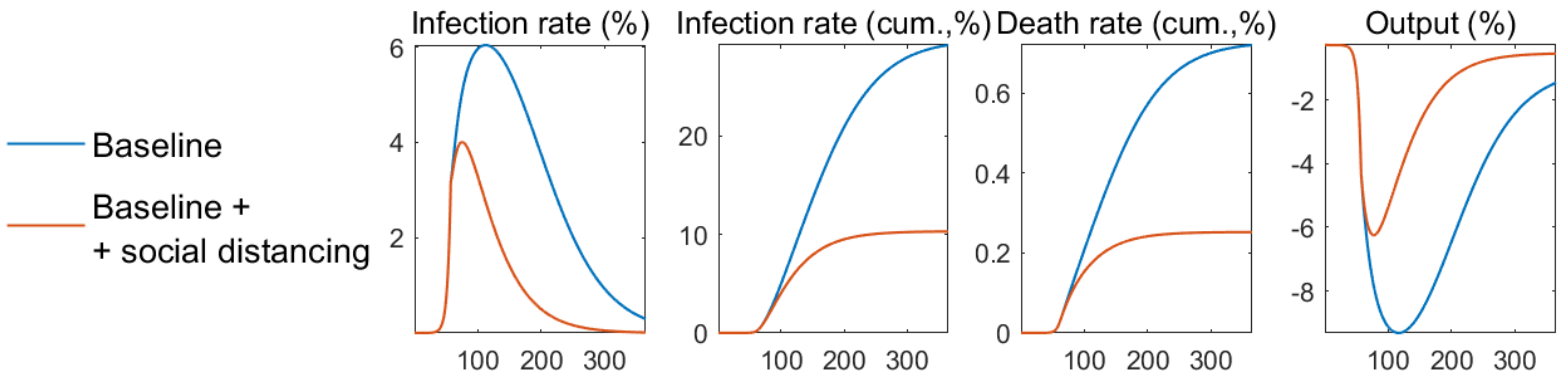

\section{Targeted Labor Market Restrictions}

Our second mitigation policy refers to targeted labor market restrictions. More specifically, under the assumption of enhanced information - e.g. due to contact tracing, widespread testing, medical checks at workplaces, etc. - infected persons are better-identified and restricted to access workplaces. Within this 
simulation we change the working shares of undiagnosed latent (UIL) from 1 to 0.5 and of undiagnosed infectious (UII) and non-infectious (UINI) from 0.5 to 0.25. The results shown in Figure 9 present significant gains in terms of both output and health outcomes. The improvements resemble quantitatively those obtained under better social distancing measures described above.

Figure 9: Mitigation policy: targeted labor market restrictions
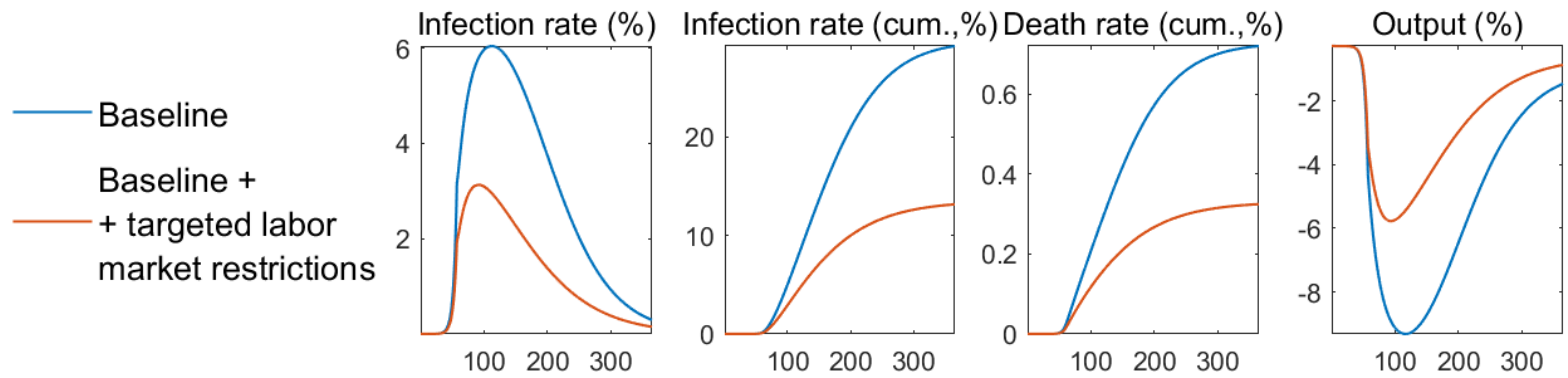

\section{Improved Quarantine and Isolation}

The third policy refers to increased quarantine and isolation capacity, complemented by more widespread testing, contact tracing and identification of positive cases. Cherif and Hasanov (2020) use a workhorse SIR model to show that universal testing and isolation is the most efficient policy to address the COVID-19 pandemic. We simulate an improved quarantine and isolation scenario by doubling the baseline quarantine fraction to $\phi_{0}=0.1$; see Figure 10. Note that given our formulation of quarantine transition, this simulation is equivalent to halving the isolation delay to $\delta=2.5$ days. The mitigation policy achieves a flattening of both infection and recession curves relative to the baseline model.

Figure 10: Mitigation policy: increased identification and quarantine
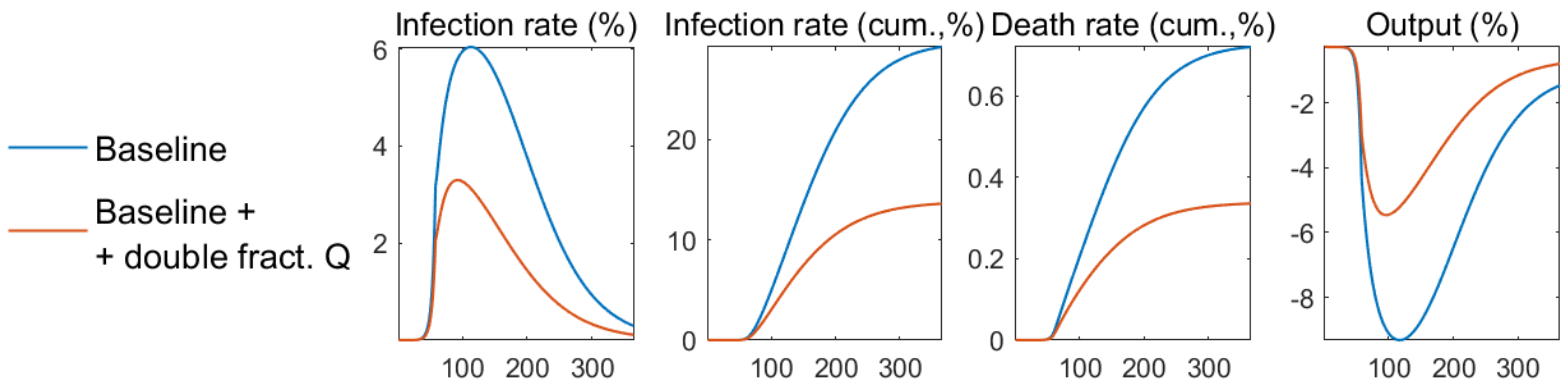

\section{E. Increasing Telework Capacity}

Finally, we simulate the effects of additional investment in telework capacity - via endowing workers with technical equipment, digitalization, etc. Technically, we impose an increase in the TFP term $(Z)$ of 2.5 percent 
over four months starting from the declaration of emergency. This level of productivity shock is chosen mostly for illustrative purposes, but can also be put in perspective by comparing it to the projected fiscal balance deterioration and gross government debt increase over 2020 relatively to 2019, assuming that a part of the underling fiscal measures will be reflected in better digital infrastructure. ${ }^{12}$ In addition, given that a proportion of workers do not need to access work facilities under this scenario, the contact frequency term in equations (2)-(3) is decreased proportionally to the change in TFP. As shown in Figure 11, as compared to the baseline simulation this policy results in less steep output decline and quicker recovery, as well as slightly better outcomes in terms of infection prevalence and death rates.

Figure 11: Mitigation policy: investment in telework capacity

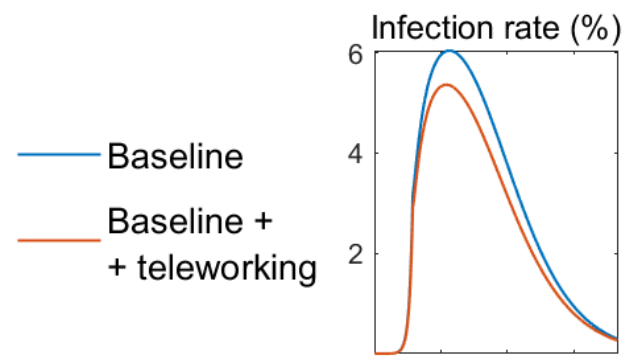

100200300

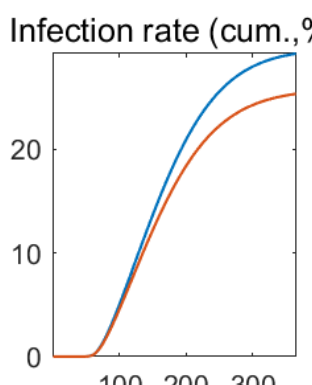

100200300

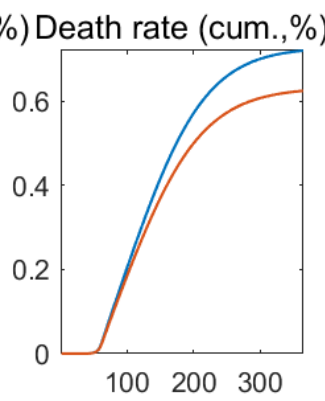

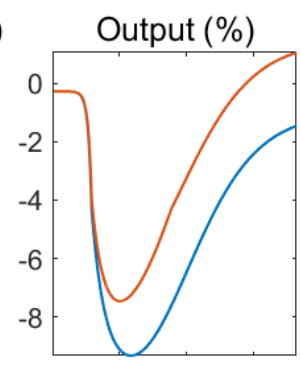

100200300

\section{F. Capturing Tradeoffs}

Overall, the simulations for various mitigation policies show how these measures flatten both the epidemic and recession curves, improving the "lives versus livelihoods" tradeoff. In order to illustrate this result, Acemoglu et al. (2020) simulate the model for different calibrations for the value of statistical life parameter. The plot of the resulted death rates and output losses - as proxies for health and economic outcomes, respectively represents the pandemic possibility frontier. In our framework, one parameter that shapes the tradeoff is the sensitivity to infection rate, $\kappa$. We simulate the baseline model calibration and the increased quarantine capacity scenario for a grid of values for $\kappa$ between 0 and 5, with a step change of 0.5 . The resulted curves represent the menus of possible choices for agents/countries, depending on their assessment of the tradeoff; see Figure 12. Entities with low death risk tolerance are situated in the north-west region: they are curtailing contacts by a lot, managing to preserve more lives but at the cost of larger output drop. Conversely, risk-taking units are situated in the south-east region, where the death rate is higher, but the output drop is milder. As shown by the outward translation of the curve under increased quarantine capacity, mitigation policies are pushing the pandemic possibility frontier towards improved outcomes and alleviate the tradeoffs.

${ }^{12}$ According to the June 2020 IMF World Economic Outlook Update, fiscal deficit (GDP share) is projected to increase from 4.9 percent in 2019 to 10.6 percent in 2020 in emerging markets economies, and from 4.1 percent to 6.1 percent in low-income developing countries. At the same time, gross government debt (GDP share) is projected to increase from 52.4 percent to 63.1 percent in the former group of countries, and from 43.1 percent to 48.2 percent in the latter. 
Figure 12: Pandemic possibility frontier

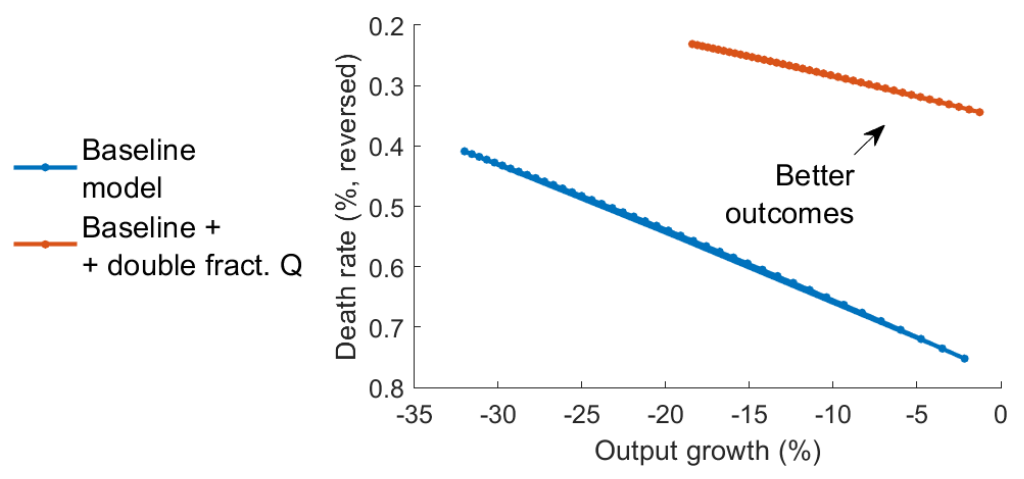

Our framework constrains us from ranking the analyzed containment policies since we do not provide a quantitative cost-benefit analysis and do not study the potential financing sources and practical implementation details. In practice, country authorities have adopted a mix of policies, with the marginal contribution of individual measures impossible to isolate. Nevertheless, our simulations provide relevant information on the likely effects across a range of stylized representations of the most widely discussed intervention policies.

\section{SECOND INFECTION WAVE, MEDICAL SOLUTIONS AND TEMPORARY IMMUNITY}

In this section we explore relevant scenarios beyond the short-term, such as the emergence of multiple infection waves, the discovery of proven medical solutions (treatment or vaccine), and the possibility of temporary (rather than permanent) immunity achieved by the recovered individuals.

\section{A. Second Infection Wave and Mitigation Policies}

The second infection wave simulation is generated by increasing the contact frequency to 2.5 (from 2) persons starting day 200, which in our timeline corresponds to early-autumn 2020. The results are presented in Figure 13, alongside the baseline model results from the previous section. Note that the simulation horizon was increased from 365 days to 600 days. The resurgence of infections leads to a prevalence of COVID-19 of more than 60 percent and a fatality rate amounting to 1.5 percent of the total population. ${ }^{13}$ The epidemic generates a double dip recession, with the average output loss of 8 percent over the first year.

\footnotetext{
${ }^{13}$ The 60 percent prevalence is used in some of the relevant literature, e.g. Eichenbaum et al. (2020), to calibrate SIR-type models.
} 
Figure 13: Second infection wave
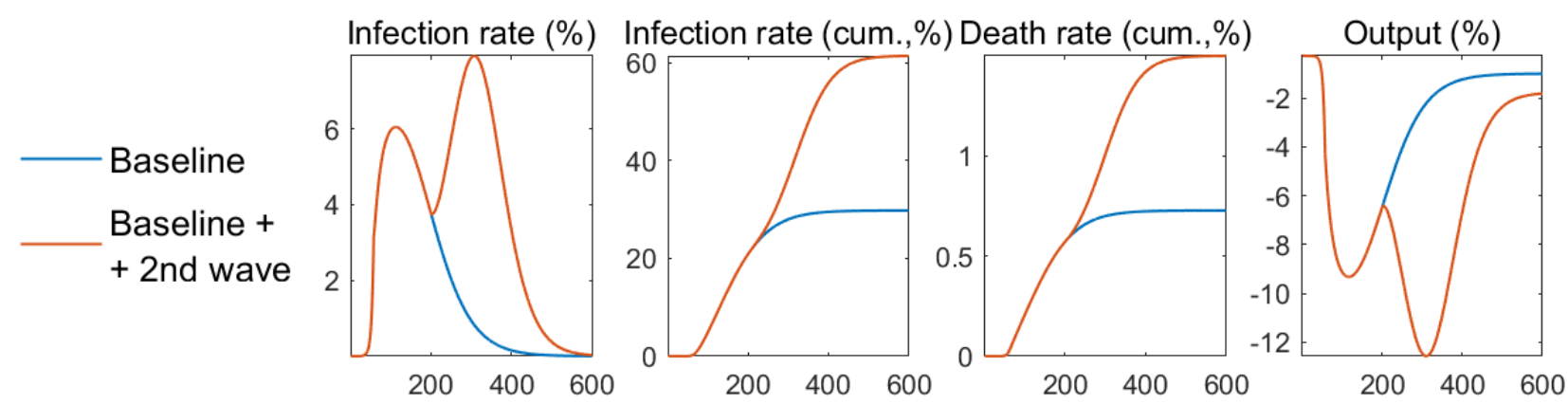

Figure 14 displays the simulation results across the four mitigation policies analyzed in the previous section. The design and calibration of containment policy scenarios is identical; note that in the case of better social distancing measures, the 15 percent reduction in effective contact frequency implies a decrease from 2.5 to 2.125 persons. Each policy is deployed after day 230, i.e. one month after the second infection wave emerges. As mentioned above, the scenarios do not include a proper cost-benefit assessment, so that a relevant ranking of the mitigation policies based on the simulations is not applicable. Overall, in relative terms, the more the measures flatten the second infection curve, the more these measures are efficient at reducing the severity of the economic contraction, reflecting the benefits of containment policies. A related interpretation of these outcomes reflects the presence of a learning mechanism taking place, which will likely make the second infection wave different from the first one. We (indirectly) consider this accumulated knowledge via the various policies being deployed and alleviating the negative outcomes of the shock. In other words, while we first present the "Baseline + 2nd wave" simulation in Figure 13, it could well be the case that the actual trajectory of the second infection wave will be one (or a combination) of the simulations from Figure 14, i.e. including some mitigation policies.

Figure 14: Mitigation policies: second infection wave
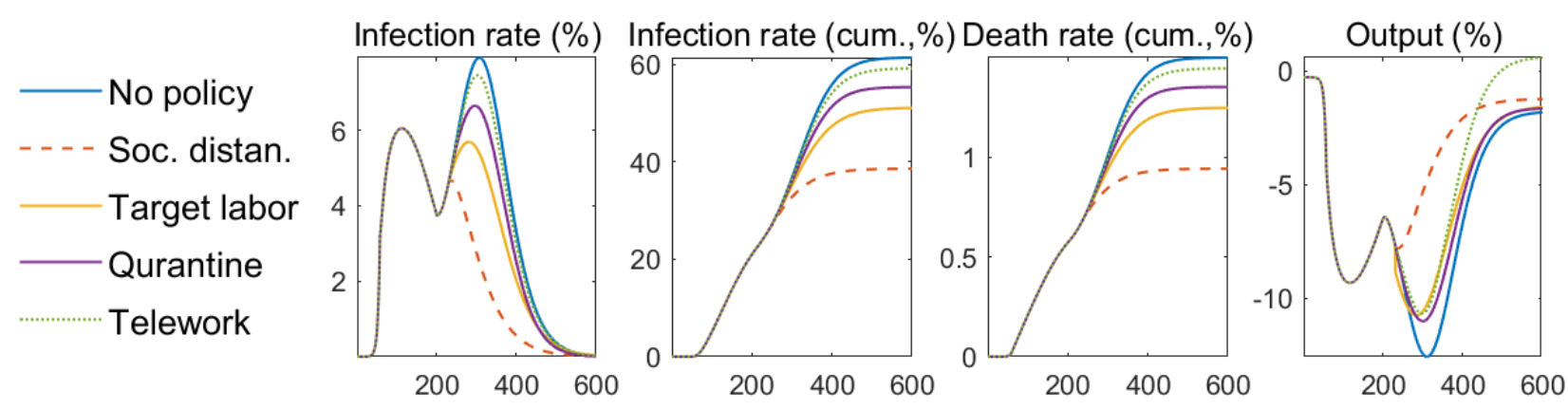

\section{B. Medical Solutions}

The arrival of proven medical solutions - either in the form of treatment or vaccine - is a much hoped-for outcome of the current scientific research efforts. We simulate the corresponding scenarios in our framework 
as follows. In case of the treatment, the mortality rate is reduced to zero, so that even if the prevalence of the disease remains elevated, all infected individuals fully recover. For a vaccine, we assume there are no more new infections and the epidemic disappears once the already infected individuals recover or die. Both solutions are assumed to arrive at day 300 (late-autumn 2020 in our timeline). The results are presented in Figure 15.

Figure 15: Treatment and vaccine
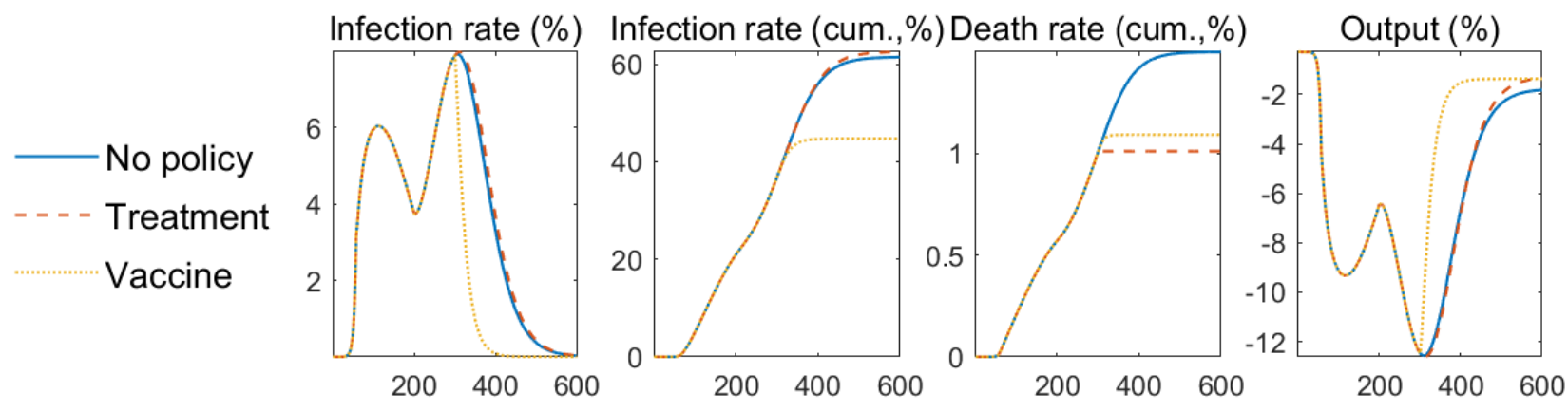

Effective treatment eliminates completely the risk of mortality. Nevertheless, given that the transmission of the virus remains, as before, the infection curve and economic outcomes are very similar to the initial scenario. In our model formulation, in the treatment scenario, the resulted infection rates are likely to be understated while the severity of the economic downturn overstated, given that under a widely available treatment (i) people are expected to change their behavior towards more interactions (equivalent to lower $\kappa$ ) and (ii) labor market access conditions across different health groups will presumably be relaxed (equivalent to higher sharework $k_{i}$ ). These changes will result in more infections but less economic damage - which under efficient treatment will not represent as stark a tradeoff as in the baseline scenario.

The discovery of the vaccine will eliminate the infection risk, and only the individuals who are already infected face the risk of dying. Once the vaccine is administered across the population and the virus is completely eradicated, output quickly rebounds to the pre-pandemic level. Besides the uncertainty related to the moment of an efficient vaccine discovery, in practice, once a company/country succeeds in developing the vaccine, it will likely take time to administer it to all the population, rendering our simulation results in Figure 15 as optimistic. Accordingly, it is important that countries collaborate and prepare efficient protocols to speed-up the production and delivery of the vaccine worldwide, once it becomes available.

\section{Temporary Immunity Consideration}

In the baseline model, we follow the relevant literature and assume that recovery is an absorbing state, i.e. achieved immunity is permanent. However, clinical assessments point to a gradual reduction of antibodies in recovered individuals; see Long et al. (2020). Accordingly, we simulate an alternative scenario in which the duration of immunity is finite, adjusting the equation for recovered individuals as: 


$$
R(t)=\psi U I N I(t)+\psi Q(t)-\frac{R(t)}{\lambda}
$$

where $\lambda$ represents the duration of immunity. Furthermore, equation (2) is amended with the same flow of individuals who are losing immunity and transition back to the susceptible pool, $\frac{R(t)}{\lambda}$.

Figure 16 shows the simulation results for $\lambda=90$ days, alongside the baseline calibration $\lambda=\infty$. Since the pool of susceptible individuals is constantly supplemented by the recovered persons who lose immunity - and in the absence of any mitigation policies or medical solutions - the prevalence of the disease is significantly higher and continues even in the long run. Accordingly, the output loss is very persistent. The temporary immunity simulation underscores the importance of efficient mitigation policies and medical research in order to avoid permanent losses in lives and livelihoods.

Figure 16: Temporary immunity

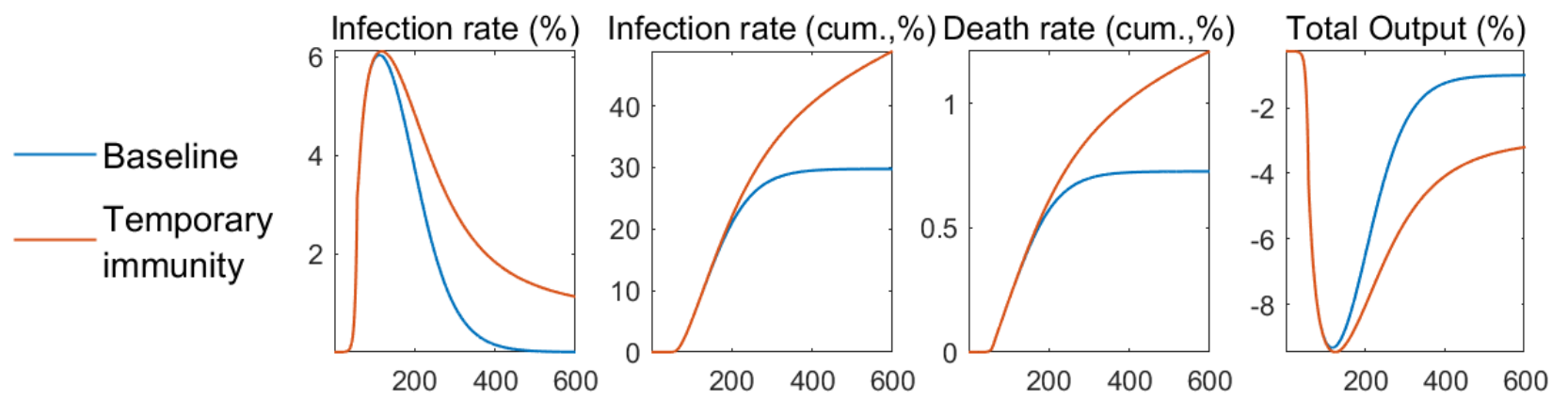

\section{MULTISECTOR EXTENSION}

Official statistical data and surveys released over the recent period point to highly asymmetric effects of the COVID-19 shock and significant reallocation of activity across different economic sectors, as detailed in Barrero et al. (2020). This evidence has inspired the development of multisector integrated epidemiologicalmacroeconomic models: Baqaee et al. (2020), Cakmakli et al. (2020), Krueger et al. (2020), etc. In this context, we exploit a similar extension to our analytical framework, with the final consumption good representing a bundle over a range of sectoral intermediate goods. The latter are produced under specific working conditions, matching the sectoral-level heterogeneity in terms of social interaction, workers' proximity and infection risk.

Let $n=1,2, \ldots, N$ index the intermediate good sectors. We assume the production function for a generic sector $n$ is similar to the one in our baseline (homogenous) model, equation (9), but with behavioral response sensitivity $\kappa_{n}$ representing the sector-specific "infection intensity" or "relative contagiousness", as in Krueger et al. (2020):

$$
Y_{n}(t)=\omega_{n} \cdot Z(t) \cdot\left[\sum_{i} i(t) \cdot \frac{W A P_{i}(t)}{i(t)} \cdot \frac{L_{i}(t)}{W A P_{i}(t)} \cdot \operatorname{sharework}_{i}(t)\right] \cdot\left[1-\kappa_{n} \cdot \tau(t)\right]
$$


where $\omega_{n}$ represents the sector share in aggregate production. For tractability, we assume the labor input is homogeneous across the sectors and the only relevant difference refers to the infection intensity $\kappa_{n}$. The final good is aggregated using a constant elasticity of substitution function:

$$
Y(t)=\left[\sum_{n} \omega_{n}^{\frac{1}{\eta}}\left(Y_{n}(t)\right)^{\frac{\eta-1}{\eta}}\right]^{\frac{\eta}{\eta-1}}
$$

where $\eta$ represents the elasticity of substitution among the intermediate goods.

For a simple illustration of the model extension, we assume an economy with two sectors $(N=2)$ of equal shares $\left(\omega_{1}=\omega_{2}=0.5\right)$, but with the first sector twice as contagious $\left(\kappa_{1}=5, \kappa_{2}=2.5\right)$ and hence affected relatively more by the pandemic developments. As shown in Figure 17, the aggregate outcomes depend on the degree of substitutability between $Y_{1}$ and $Y_{2}$. In particular, total output declines more when the intermediate goods are complements $(\eta=0.5)$ as compared to substitutes $(\eta=3)$. This result can be explained as follows: when sectoral goods can be easily substituted (higher $\eta$ ), the loss in production capacity for the more affected sector $\left(Y_{1}\right)$ can be partly compensated by an increase in economic activity in the less affected sector $\left(Y_{2}\right)$. Conversely, if the intermediate goods cannot be easily substituted and need to be consumed together (lower $\eta$ ), activity in both sectors will be declining by relatively more. As such, substitutability of sectoral production acts as a shock absorber in terms of aggregate output. However - in line with the outcomes reported in Section III - absent mitigation policies, a lower output loss implies more widespread infection and higher fatality rates.

Figure 17: Multisector extension: equal sectoral weights
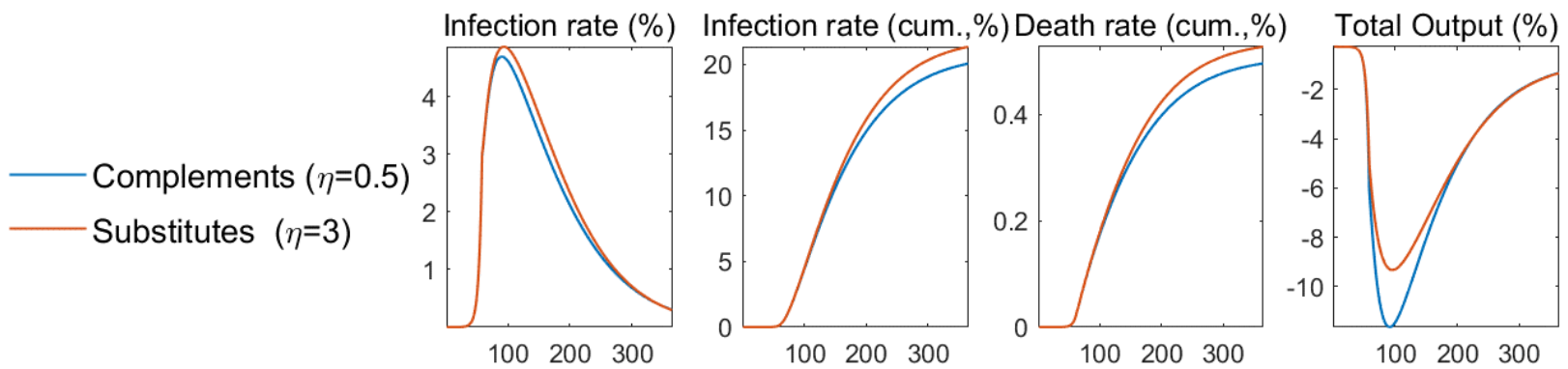

The results are sensitive to the relative importance of the individual sectors. For example, Figure 18 displays the simulation results for an economy with highly concentrated economic activity in the more affected sector $\left(\omega_{1}=0.75, \omega_{2}=0.25\right)$. In this case, substitutability provides only a limited buffer, so that total output decreases by more than in the equal-weights calibration above, but with small relative gains in terms of lower infections and deaths. 
Figure 18: Multisector extension: affected sector more important
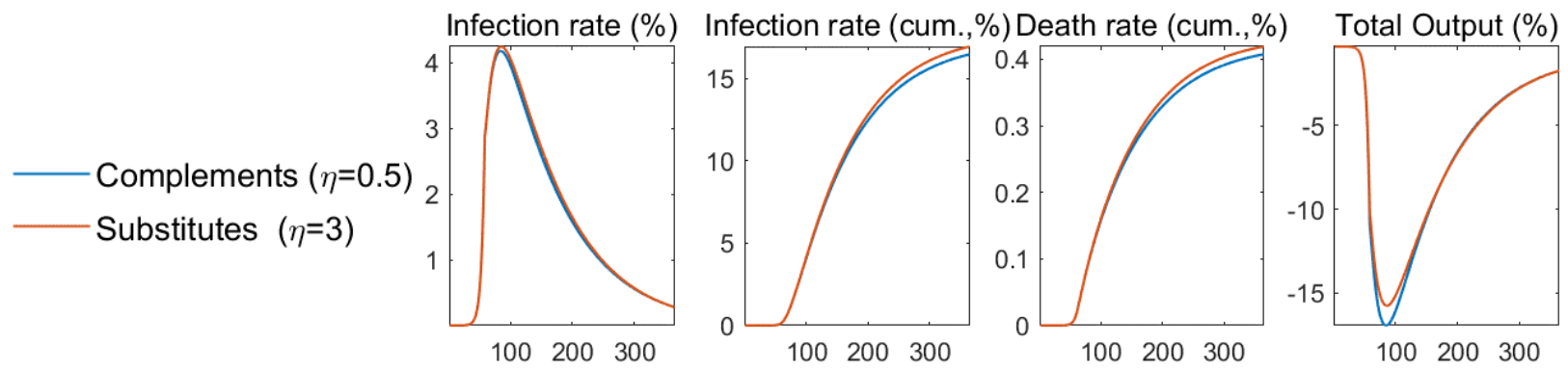

The results illustrated above suggest the relevance of sector-specific policies, e.g. lockdown restrictions targeted toward more infectious activities/occupations, particularly when there is substitutability in production (or consumption) across the sectors; the implementation of these policies in the model is left for future research. The analytical framework can be extended further to more than two sectors, e.g. to match actual decomposition in terms of primary, secondary and tertiary economic activities. Also, it can allow for a more meaningful differentiation in terms of production technologies - e.g. by adjusting upward the shares of individuals across health states that have access to the labor market if they are employed in "essential sectors".

\section{CONCLUSION}

In this paper we develop a flexible analytical framework to analyze the interaction and spillover effects between the epidemiological aspects of COVID-19 and macroeconomic outcomes. The model can be calibrated to generic EMDEs, which generally have limited healthcare and financial resources, reduced telework capacity, limited precautionary savings and high levels of informality. The multiplicity of health states in the model especially the differentiation between latent, infectious, and non-infectious stages for the infected individuals - allows simulating epidemiological and economic outcomes related to a variety of representative mitigation policies. Accordingly, our results provide a broad assessment of the options to alleviate the tradeoff between saving lives and preserving economic outcomes policymakers currently face.

Our framework encompass relevant attributes and channels: the imposition of government-mandated lockdowns and an endogenous mechanism that links population compliance with the lockdown measures to current economic conditions - which is likely to be an important channel in the case of EMDEs; voluntary changes in population behavior whereby individuals internalize the infection risk; the development of efficient medical solutions in the form of treatment or vaccine. We also explore the intersectoral reallocation effects the COVID-19 shock is entailing by extending the model to a multisector setting.

Model simulations provide relevant information for decision-making about the effects across a range of stylized representations of the most prominent intervention policies discussed or implemented worldwide. The framework can also be a useful tool in teaching and capacity building activities. The results underscore the benefits in terms of both minimizing adverse health-related outcomes and improving economic activity. 
Additional scenarios demonstrate that efficient deployment and propagation of containment policies become especially important in the case of repeated infection waves or limited immunity for the recovered patients.

\section{REFERENCES}

Acemoglu, D., Chernozhukov, V., Werning, I., \& Whinston M. D. (2020) A Multi-Risk SIR Model with Optimally Targeted Lockdown. NBER Working Paper No. 27102.

Alon, T., Kim, M., Lagakos, D. \& VanVuren, M. (2020) How Should Policy Responses to the COVID-19 Pandemic Differ in the Developing World? NBER Working Paper No. 27273.

Alvarez, F. E., Argente, D., \& Lippi F. (2020) A Simple Planning Problem for COVID-19 Lockdown. NBER Working Paper No. 26981.

Baqaee, D., Farhi, E., Mina, M., \& Stock, J. H. (2020) Policies for a second wave. Brookings Papers on Economic Activity Conference Drafts.

Barrero, J. M., Bloom, D., \& Davis, S. (2020) COVID-19 is also a reallocation shock. Brookings Papers on Economic Activity Conference Drafts.

Brussevich, M., Dabla-Norris, E., \& Khalid, S. (2020) Who will Bear the Brunt of Lockdown Policies? Evidence from Tele-workability Measures Across Countries. IMF Working Paper 20/88.

Cakmakli, C., Demiralp, S., Kalemli-Ozcan, S., Yesiltas, S., \& Yildirim M. (2020) COVID-19 and Emerging Markets: An Epidemiological Model with International Production Networks and Capital Flows. IMF Working Paper 20/133, International Monetary Fund.

Chen, S., Igan, D., Pierri, N., \& Presbitero, A. (2020) Tracking the Economic Impact of COVID-19 and Mitigation Policies in Europe and the United States. IMF Working Paper 20/125, International Monetary Fund.

Cherif, R., \& Hasanov, F. (2020) A TIP Against the COVID-19 Pandemic. IMF Working Paper 20/114, International Monetary Fund.

Chu, D. K., Akl, E. A., Duda, S., Solo, K., Yaacoub, S., \& Schünemann, H. J. (2020) Physical distancing, face masks, and eye protection to prevent person-to-person transmission of SARS-CoV-2 and COVID-19: a systematic review and meta-analysis. The Lancet, 395 (10242), 1973-1987.

Chudik, A., Pesaran H., \& Rebucci A. (2020) Voluntary and Mandatory Social Distancing: Evidence on COVID-19 Exposure Rates from Chinese Provinces and Selected Countries. CESifo Working Paper No. 8243.

Correia, S., Luck, S., \& Verner, E. (2020) Pandemics Depress the Economy, Public Health Interventions Do Not: Evidence from the 1918 Flu (June 5, 2020). Available at SSRN: https://ssrn.com/abstract=3561560.

Cox, N., Ganong, P., Noel, P., Vavra, J., Wong, A., Farrell, D., \& Greig, F. (2020) Initial impacts of the pandemic on consumer behavior: Evidence from linked income, spending, and savings data. Brookings Papers on Economic Activity Conference Drafts. 
Deb, P., Furceri, D., Ostry, J., \& Tawk, N. (2020) The effect of containment measures on the COVID-19 pandemic. Covid Economics, CEPR Vetted and Real-Time Papers, Issue 19.

Eichenbaum, M., Rebelo, S., \& Trabandt, M. (2020) The Macroeconomics of Epidemics. NBER Working Paper No. 26882.

Eksin, C., Paarporn, K., \& Weitz J. S. (2019) Systematic biases in disease forecasting - The role of behavior change. Epidemics 27, 96-105.

Goldberg, P. K., \& Reed, T. (2020) The effects of the coronavirus pandemic in emerging markets and developing economies: An optimistic preliminary account. Brookings Papers on Economic Activity Conference Drafts.

Gupta, S., Simon, K., \& Wing, C. (2020) Mandated and voluntary social distancing during the COVID-19 epidemic. BPEA Conference Drafts.

He, X., Lau, E. H. Y., Wu, P., Deng, X., Wang, J., Hao, X., Lau, Y. C., Wong, J. Y., Guan, Y., Tan, X., Mo, X., Chen, Y., Liao, B., Chen, W., Hu, F., Zhang, Q., Zhong, M., Wu, Y., Zhao, L., Zhang, F., Cowling, B. J., Li F., \& Leung, G. (2020) Temporal dynamics in viral shedding and transmissibility of COVID-19. Nature Medicine 26, 672-675.

International Monetary Fund (2020) Monetary and Financial Policy Responses for Emerging Market and Developing Economies. IMF Special Series on COVID-19.

Kalemli-Ozcan, S. (2020) Are Macro and Credit Policies Enough? IMF Special Series on COVID-19.

Kermack, W. O., \& McKendrick, A. G. (1927) A Contribution to the Mathematical Theory of Epidemics. Proceedings of the Royal Society of London, series A 115, 772, 700-721.

Krueger, D., Uhlig, H., \& Xie, T. (2020) Macroeconomic Dynamics and Reallocation in an Epidemic. NBER Working Paper No. 27047.

Loayza, N. (2020) Costs and Trade-Offs in the Fight against the COVID-19 Pandemic: A Developing Country Perspective. World Bank Group Research \& Policy Briefs No. 35.

Long, Q., Tang, X., Shi, Q., Li, Q., Deng, H., Yuan, J., Hu, J., Xu, W., Zhang, Y., Lv, F., Su, K., Zhang, F., Gong, J., Wu, B., Liu, X., Li, J., Qiu, J., Chen, J., \& Huang A. (2020) Clinical and immunological assessment of asymptomatic SARS-CoV-2 infections. Nature Medicine Letters.

Maloney, W., \& Taskin, T. (2020) Determinants of Social Distancing and Economic Activity during COVID19: A Global View. World Bank Group Policy Research Working Paper 9242.

National Centre for Infectious Diseases (2020) Position Statement from the National Centre for Infectious Diseases and the Chapter of Infectious Disease Physicians, Academy of Medicine, Singapore - 23 May 2020. 